\title{
Quantitative phase imaging of live cells with near on-axis digital holographic microscopy using constrained optimization approach
}

\author{
Vimal Prabhu Pandiyan \\ Kedar Khare \\ Renu John
}




\title{
Quantitative phase imaging of live cells with near on-axis digital holographic microscopy using constrained optimization approach
}

\author{
Vimal Prabhu Pandiyan, ${ }^{a}$ Kedar Khare, ${ }^{\mathrm{b}, *}$ and Renu John $n^{\mathrm{a}, *}$ \\ ${ }^{a}$ Indian Institute of Technology Hyderabad, Department of Biomedical Engineering, Kandi, Sangareddy, Telangana 502285, India \\ ${ }^{b}$ Indian Institute of Technology Delhi, Department of Physics, Hauz Khas, New Delhi 110 016, India
}

\begin{abstract}
We demonstrate a single-shot near on-axis digital holographic microscope that uses a constrained optimization approach for retrieval of the complex object function in the hologram plane. The recovered complex object is back-propagated from the hologram plane to image plane using the Fresnel back-propagation algorithm. A numerical aberration compensation algorithm is employed for correcting the aberrations in the object beam. The reference beam angle is calculated automatically using the modulation property of Fourier transform without any additional recording. We demonstrate this approach using a United States Air Force (USAF) resolution target as an object on our digital holographic microscope. We also demonstrate this approach by recovering the quantitative phase images of live yeast cells, red blood cells and dynamics of live dividing yeast cells. (C) 2016 Society of Photo-Optical Instrumentation Engineers (SPIE) [DOI: 10.1117/1.JBO.21.10.106003]
\end{abstract}

Keywords: digital holographic microscopy; quantitative phase imaging; constrained optimization approach.

Paper 160267PRR received Apr. 23, 2016; accepted for publication Sep. 29, 2016; published online Oct. 21, 2016.

\section{Introduction}

Biological specimens are weakly scattering and absorbing and hence it is hard to visualize them using a bright field microscope. Zernike's phase contrast microscope ${ }^{1}$ solves this problem by converting the phase change of the object into amplitude change to improve contrast. But quantitative estimation of phase is not feasible with this technique. Wang et al. developed a new technique named as spatial light interference microscopy ${ }^{2}$ combining the principle of Zernike phase contrast microscopy and temporal phase shifting ${ }^{3}$ to quantify the phase, but fast imaging is limited with this technique due to its multiple recording nature. Single-shot quantitative phase imaging (QPI) techniques such as Hilbert phase microscopy ${ }^{4}$, digital holographic microscopy (DHM) ${ }^{5,6}$ diffraction phase microscopy (DPM), ${ }^{7,8}$ and white light diffraction phase microscopy (w-DPM) have been developed in the last few decades, aiming at visualizing weakly scattering and absorbing biological specimens with high contrast and quantifying the thickness of the specimens to subwavelength accuracies. These QPI techniques have been explored in different biological applications. ${ }^{9-12}$ Ikeda et al. developed the Hilbert phase microscopy and demonstrated its application in studying the morphology of red blood cells (RBCs), quantifying the $\mathrm{RBC}$ volume, observing the thermal fluctuations in RBCs, and imaging tissue slices of mouse brain, spleen, and liver. ${ }^{4,13,14}$ DHM has been demonstrated in various applications such as quantifying the thickness of living neuron cells, ${ }^{15}$ imaging the remodeling of synaptic network in neurons, ${ }^{11}$ sperm imaging, ${ }^{16,17}$ and so on. Park et al. ${ }^{7}$ have combined the diffraction phase and fluorescence microscopy and demonstrated its application in simultaneous quantitative phase image and epifluorescence imaging of a mitotic kidney cell. Bhaduri et al. ${ }^{18}$ have

*Address all correspondence to: Kedar khare, E-mail: kedar@ physics.iitd.ac.in Renu John, E-mail: renujohn@iith.ac.in developed the w-DPM and shown its application in imaging cell structure of HeLa cells with nucleoli. Most of the above techniques use the off-axis interferometric principle and hence require the reference beam to be at a minimum angle ${ }^{19}$ to spatially separate the DC and twin image terms in the Fourier domain. Few techniques use spatial phase shifting methods to separate the DC and twin image terms. ${ }^{20,21}$ However, phase shifting should be optimized to separate completely, the DC and the twin images for reconstruction. In the case of off-axis interferometric imaging, these spatial frequencies are often filtered using Fourier filtering techniques. ${ }^{22}$ The minimum reference angle required to reconstruct the off-axis interferograms of the object with the highest spatial frequency $f_{x}$ is given by $\sin ^{-1}\left(3 f_{x} \lambda\right) .{ }^{19}$ With commercially available cameras, with typical pixel sizes of 2 to $5 \mu \mathrm{m}$ in the visible wavelength region, this minimum angle criterion limits the achievable spatial resolution of the object. In other words, these off-axis interferometric techniques do not utilize the full spatial resolution offered by digital cameras $^{23}$ and hence the practical resolution limit is well below the actual optical diffraction limit. This problem is addressed in temporal phase shifting interferometric (PSI) techniques ${ }^{24}$ in which multiple on-axis interferograms are recorded at different phase steps and the object phase can be retrieved with high spatial resolution. But due to the multiple recording nature, PSI prevents the real-time dynamic imaging.

Recently, a constrained optimization approach has been described to address this reconstruction process in digital holography. ${ }^{25,26}$ Experimental results show excellent high-resolution image recovery compared to conventional Fourier filtering techniques, for the interferograms recorded with less than minimum angle criterion. This constrained optimization approach has been demonstrated to image the RBCs using DHM at

$1083-3668 / 2016 / \$ 25.00$ @ 2016 SPIE 
the image plane. ${ }^{27}$ However, it requires an additional recording of the hologram to find the angle of reference beam. It also requires an additional microscope objective (MO) in the reference arm that is identical to the one used in the object arm to correct the aberrations introduced in the object arm.

In this paper, we use the modulation property of the Fourier transform to find the reference beam angle from the hologram without any additional recording. A numerical aberration compensation algorithm is employed for correcting the aberrations in the object beam. The complex object field is recovered at the hologram plane by solving a constrained optimization problem. The optimization problem is solved by a gradient descent approach iteratively and a smoothness constraint is implemented by spatial averaging with an appropriately sized window. The recovered complex object is back-propagated from the hologram plane to the image plane using the Fresnel back-propagation algorithm. Experimental results show a higher resolution for image recovery of a United States Air Force (USAF) resolution target with the constrained optimization approach compared to the conventional Fourier filtering technique. We also demonstrate the potential of this technique in imaging biological cells, by reconstructing high-resolution quantitative phase microscope images of yeast cells, RBCs, and the dynamics of live dividing yeast cells.

\section{Experimental Set-Up}

A Mach-Zehnder geometry is used to design the basic near onaxis DHM experimental set-up (Fig. 1). A coherent green laser source (wavelength: $532 \mathrm{~nm}$, power $10 \mathrm{~mW}$, Coherent ${ }^{\mathrm{TM}}$ make) is split into two beams using a beam splitter, to be used as the object, and the reference beams. Both the beams are spatial filtered and expanded using a spatial filter-beam expander assembly. The object beam passes through the specimen and is magnified using an MO. The magnified object wave interferes on the detector array with the reference beam $(R)$ to produce the digital hologram of the microscopic object in a near on-axis configuration. In this near on-axis DHM configuration, the reference beam is used at a very small angle and the angle is not sufficient to spatially resolve the DC and twin images. It is always inconvenient to have a large reference beam angle while performing high-resolution microscopy of live cells and biological specimens due to the limited higher spatial frequencies recording by the camera. ${ }^{28}$ However, the reconstructed image is poorly resolved, if the hologram is recorded below a minimum reference angle. In this experiment, we explicitly record the hologram with the reference beam below its minimum reference

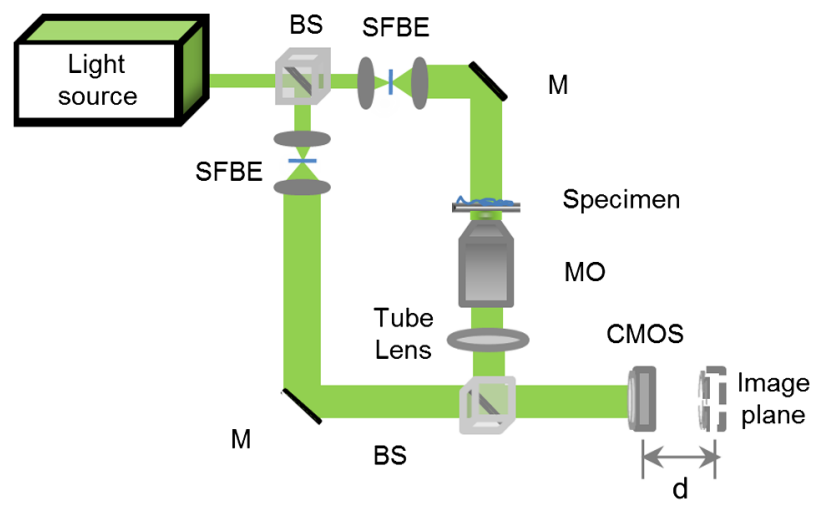

Fig. 1 Schematic representation of the experimental set-up for a digital holographic microscope (SFBE: spatial filter and beam expander; M: mirror; MO: microscope objective; BS: beam splitter.) beam angle to show the capability of our approach. A CMOS (IDS, UI-1482LE-M, $2560 \times 1920$ pixel array $2.2 \mu \mathrm{m}$ square pixel) array records the digital hologram $4 \mathrm{~cm}$ away from the image plane on the microscope and it is transferred to the computer for further processing. Image reconstructions are carried out on a MATLAB R2015 platform.

\section{Reconstruction Process}

The recovery of complex object information from a single digital hologram is modeled as a constrained optimization problem. ${ }^{25,26,29}$ The hologram $H(x, y)$ recorded due to the interference between the object beam $O(x, y)$ and the reference beam $R(x, y)$ at the hologram plane is given by

$$
\begin{aligned}
H(x, y)= & |O(x, y)|^{2}+|R(x, y)|^{2}+O(x, y) R^{*}(x, y) \\
& +R(x, y) O^{*}(x, y) .
\end{aligned}
$$

The complex object function $O(x, y)$ can be recovered from the hologram $H(x, y)$ with the known reference beam $R(x, y)$ using the constrained optimization approach. The known plane reference beam should be numerically coded and it should match exactly the experimental reference beam.

\subsection{Reference Beam Angle Calculation}

The plane reference beam $R(x, y)$ can be mathematically expressed as

$R(x, y)=A \exp \left[i 2 \pi / \lambda\left(k_{x} x+k_{y} y\right)\right]$

where $k_{x}, k_{y}$ are the two components of the reference wave vector and $A$ is the amplitude of the reference plane. The values of the $k_{x}$ and $k_{\mathrm{y}}$ are calculated by the modulation property of the Fourier transform of the hologram without any additional recording. If $\mathfrak{s}\{f(x, y)\}$ and $F\left(\xi_{1}, \xi_{2}\right)$ is a Fourier transform pair, then the modulation property of the Fourier transform ${ }^{30}$ is given as

$$
\exp \left[ \pm j 2 \pi\left(k_{x} x+k_{y} y\right)\right] f(x, y) \underset{\mathfrak{J}^{-1}}{\stackrel{\mathfrak{I}}{\leftrightarrows}} F\left(\xi_{1} \mp k_{x}, \xi_{2} \mp k_{y}\right) .
$$

Fourier transform of the hologram will give the DC and the sideband frequencies in the Fourier plane. By knowing the shift in the number of pixels of sideband frequencies from the DC, the values of $k_{x}$ and $k_{y}$ can be calculated as

$$
\begin{aligned}
& k_{x}=\lambda m f_{x}, \\
& k_{y}=\lambda n f_{y},
\end{aligned}
$$

where $\lambda$ is the wavelength of the light, $m$ and $n$ are the shift in the number of pixels of sideband frequencies from the DC in the $x$ - and $y$-directions, respectively, and $f_{x}$ and $f_{y}$ are spatial frequencies given by

$$
\begin{aligned}
& f_{x}=\frac{1}{M \Delta x}, \\
& f_{y}=\frac{1}{N \Delta y},
\end{aligned}
$$

where $M$ and $N$ are the number of pixels of digital array and $\Delta x$ and $\Delta y$ are the pixel sizes. 


\subsection{Constrained Optimization Approach for Single-Shot Holographic Microscopy}

The complex object function $O(x, y)$ in the hologram plane can be recovered by solving an optimization problem with a smoothness constraint. We define a cost function $C\left(O, O^{*}\right)$ to be minimized using the recorded hologram $H(x, y)$, the unknown object function $O(x, y)$ and the known reference beam $R(x, y)$

$$
\begin{aligned}
C\left(O, O^{*}\right)= & \frac{1}{2}\left\|H-\left(|O|^{2}+|R|^{2}+O R^{*}+O^{*} R\right)\right\|^{2} \\
& +\beta \mu\left(O, O^{*}\right),
\end{aligned}
$$

where the first term represents the L2-norm error and $\mu\left(O, O^{*}\right)$ is a penalty function for imposing the smoothness constraint on the complex object function $O(x, y)$. The smoothness constraint is used to reduce the effect of the DC and the twin image in the solution of the minimization problem for $O(x, y)$. The cost function in Eq. (6) is minimized using a gradient descent approach. ${ }^{21}$

The complex gradient of the cost function with respect to $O^{*}$ is represented as

$$
\begin{aligned}
\nabla_{O^{*}} C\left(O, O^{*}\right)= & -\left[H-\left(|O|^{2}+|R|^{2}+O R^{*}+O^{*} R\right)\right] \\
& \cdot(O+R)+\beta \nabla_{O^{*}} \mu\left(O, O^{*}\right) .
\end{aligned}
$$

The object function is iteratively updated by

$O^{(n+1)}=O^{(n)}-\alpha\left[\nabla_{O^{*}} C\right]_{O=O^{(n)}}$.

Here, $\nabla$ is the gradient cost function as per Eq. (7) and $\alpha$ is the positive constant denoting the step size in the negative gradient direction that is selected by the standard line-search method in each iteration. This algorithm is implemented iteratively by setting $\beta=0$ in Eq. (7), calculating the L2 norm error and updating the guess solution by Eq. (8). The smoothness constraint is then applied with an averaging square shape filter $(G)$ in each iteration. The overall algorithm is summarized into a single equation as Eq. (9) and also in Fig. 2,

$$
\begin{aligned}
O^{(n+1)}= & G \otimes\left\{O^{(n)}+\alpha\left[H-\left(\left|O^{(n)}\right|^{2}+|R|^{2}+O^{(n)} R^{*}\right.\right.\right. \\
& \left.\left.\left.+O^{(n) *} R\right) \times\left(O^{(n)}+R\right)\right]\right\} .
\end{aligned}
$$

The averaging square shape filter is chosen in such a way that there should be minimal loss in the resolution of an image after reconstruction. However, this averaging filter may have a minor effect on sharp edges if the complex object field is recovered at the image plane and hence the hologram is recorded and the complex object field is recovered at minimum $d$ distance from the image plane and then back propagates to the image plane. Also, the advantage of the numerical propagation allows to computationally refocus the slightly defocused image to the exact image plane.

\subsection{Fresnel Back Propagation}

Once the complex object function is recovered at the hologram plane then it is back-propagated ${ }^{31}$ to the image plane by the Fresnel back-propagation method

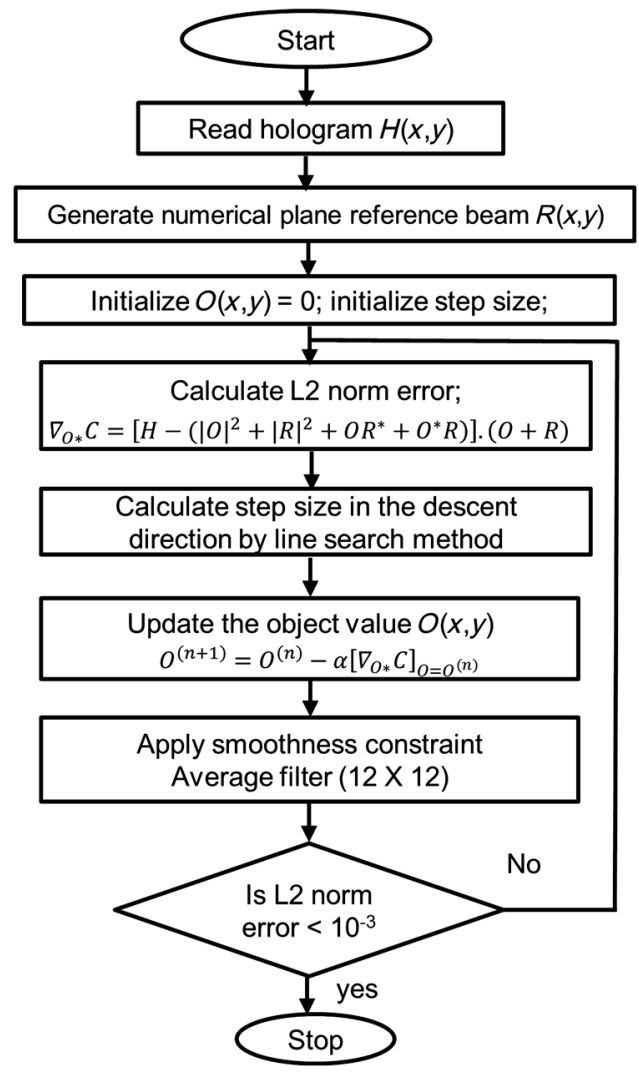

Fig. 2 Flowchart of constrained optimization approach.

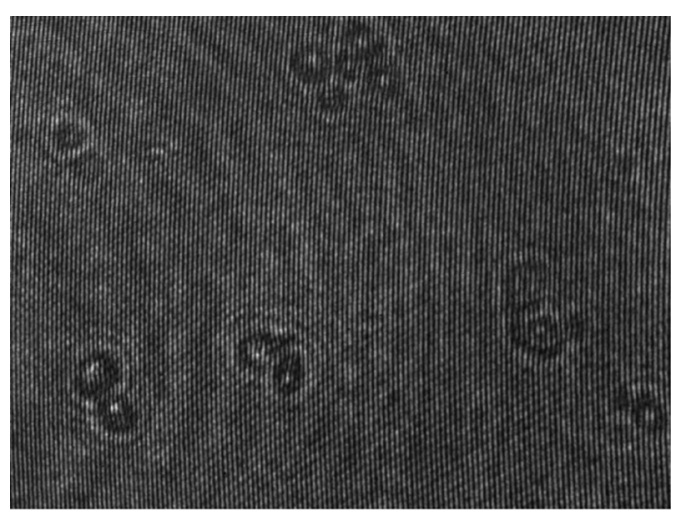

(a)

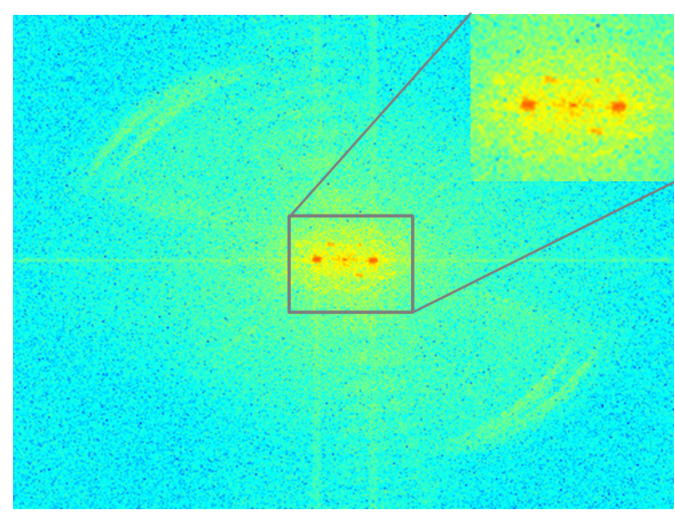

(b)

Fig. 3 (a) Hologram of yeast cells and (b) amplitude of Fourier transform of the hologram. 


$$
\begin{aligned}
\psi(m, n)= & A \exp \left[i \frac{\pi}{\lambda d}\left(m^{2} \Delta \xi^{2}+n^{2} \Delta \eta^{2}\right)\right] \\
& \times \operatorname{DFT}\left\{O(k, l) \exp \left[i \frac{\pi}{\lambda d}\left(k^{2} \Delta x^{2}+l^{2} \Delta y^{2}\right)\right]\right\}
\end{aligned}
$$

$\Delta \xi=\Delta \eta=\lambda d / N \Delta x$

where $O(k, l)$ is the recovered complex object using the constrained optimization approach in the hologram plane, $\Delta x$ and $\Delta y$ are the sampling intervals of the hologram with the CMOS camera, $d$ is the distance between the hologram plane and image plane, and $\Delta \xi$ and $\Delta \eta$ are the sampling intervals in the reconstruction plane given by Eq. (11). The reconstructed object $\psi$ in the image plane is calculated by multiplying $O(k, l)$ with $\exp \left[i(\pi / \lambda d)\left(k^{2} \Delta x^{2}+l^{2} \Delta y^{2}\right)\right]$ and applying an inverse discrete Fourier transform to the product.

\subsection{Amplitude and Phase Contrast Image}

$\psi(m, n)$ is an array of complex numbers, from which the amplitude-contrast image can be obtained by calculating the intensity given by

$$
I(m, n)=\operatorname{Re}[\psi(m, n)]^{2}+\operatorname{Im}[\psi(m, n)]^{2},
$$

and the phase image, by calculating the argument given by

$$
\phi(m, n)=\arctan \left\{\frac{\operatorname{Im}[\psi(m, n)]}{\operatorname{Re}[\psi(m, n)]}\right\} \text {. }
$$

\subsection{Automated Aberration Correction}

MO used in the object path for magnification produces a curvature aberration in the object beam. This deformation affects only the phase of the object wave and does not disturb amplitude-contrast imaging. Phase aberration can be corrected by multiplication

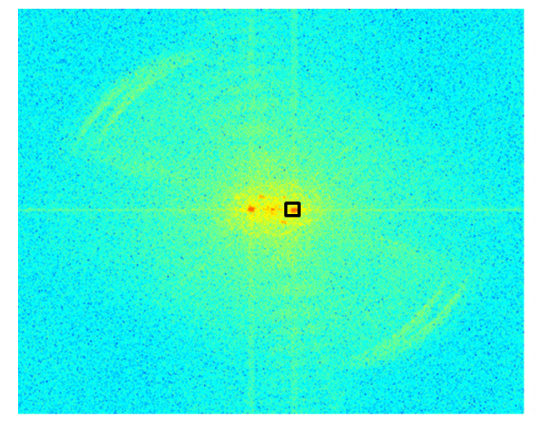

(a)

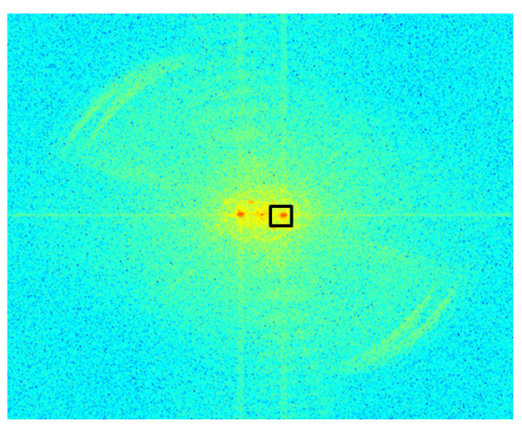

(d)

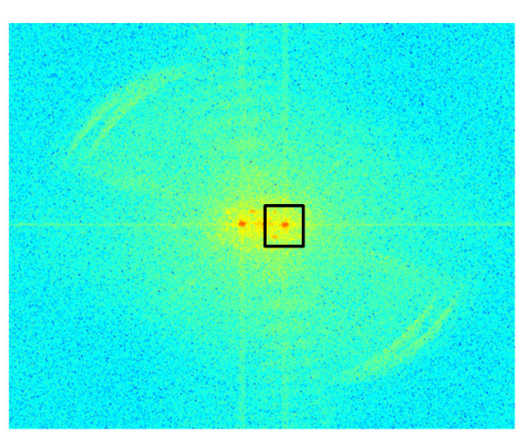

(g)

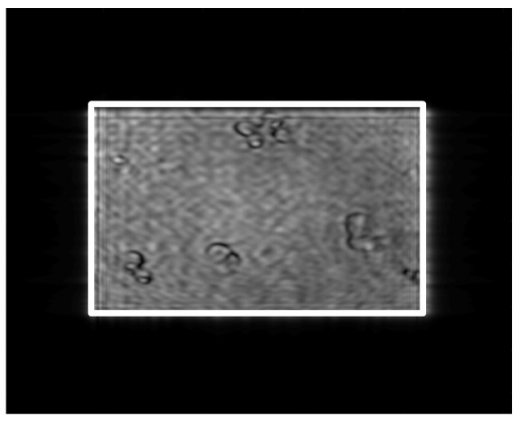

(b)

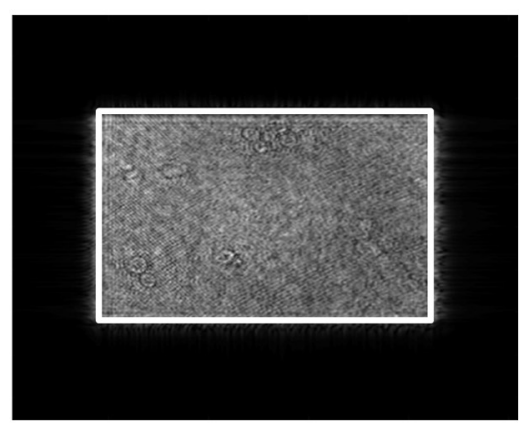

(e)

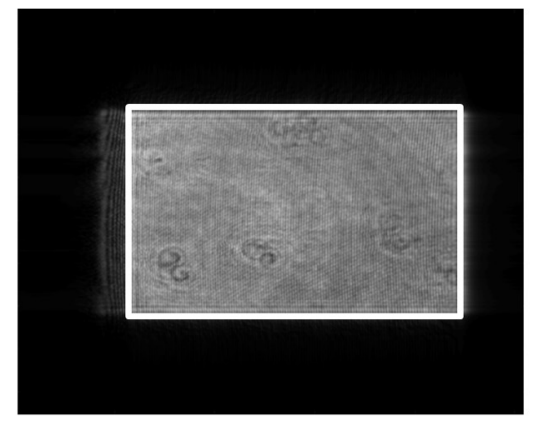

(h)

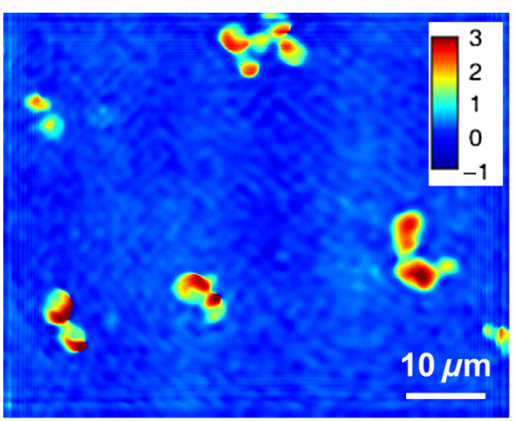

(c)

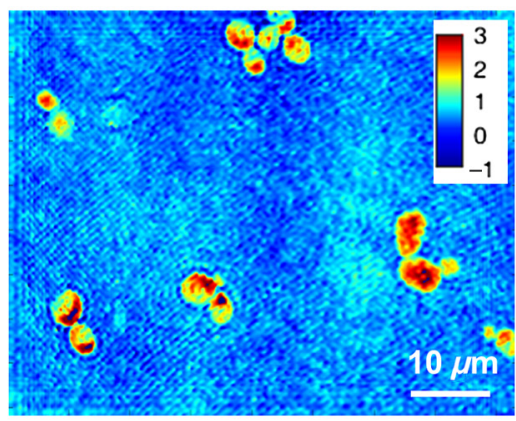

(f)

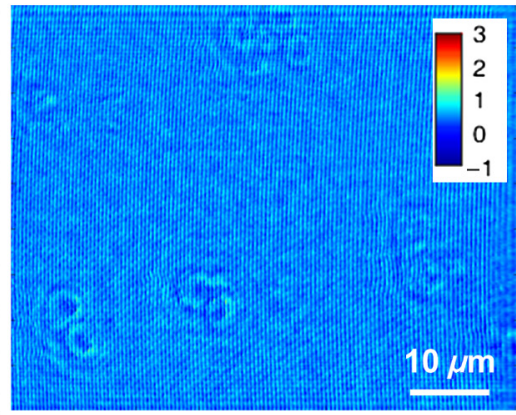

(i)

Fig. 4 Image reconstruction using Fourier filtering technique with the increasing size of filter window. (a), (d), and (g): amplitude of the Fourier transform of the hologram with square filter. (b), (e), and (h) The reconstructed amplitude images. (c), (f), and (i) The reconstructed phase images. As the filter size increases from $(40 \times 40)$ to $(120 \times 120)$ pixels, the resolution improves but it has interference from DC [Figs. 4(e) and 4(f)]. Similary as the filter size increases to $240 \times 240$ pixels, the image contains both $\mathrm{DC}$ and $\mathrm{OR}^{*}[(\mathrm{~h})$ and $(\mathrm{i})]$. (Color bar indicates phase in radians.) 
of the reconstructed wave front with the array of complex numbers called digital phase mask $\Gamma(m, n)^{31}$ and the final reconstruction equation with aberration correction is given by

$$
\begin{gathered}
\psi(m, n)=A \Gamma(m, n) \exp \left[i \frac{\pi}{\lambda d}\left(m^{2} \Delta \xi^{2}+n^{2} \Delta \eta^{2}\right)\right] \\
\times \operatorname{DFT}\left\{O(k, l) \exp \left[i \frac{\pi}{\lambda d}\left(k^{2} \Delta x^{2}+l^{2} \Delta y^{2}\right)\right]\right\} .
\end{gathered}
$$

The aberration in the system is obtained by finding the coefficients of the Zernike polynomial using phase data. ${ }^{32-34}$ The coefficients of the Zernike polynomial is calculated by the two-dimensional linear least square fitting algorithm of any order $O$ to the phase data extracted along two-dimensional profiles in the areas of the specimen known to be flat. Using these coefficients, a digital phase mask $\Gamma(m, n)$ which is the complex conjugation of aberration introduced in the object wave is calculated by

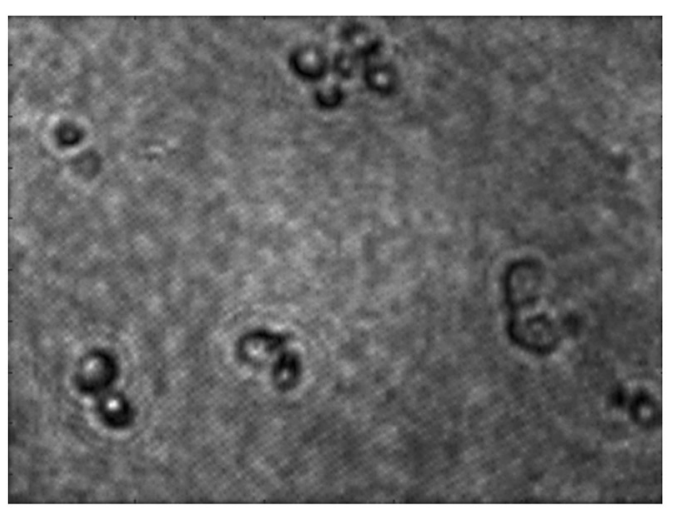

(a)

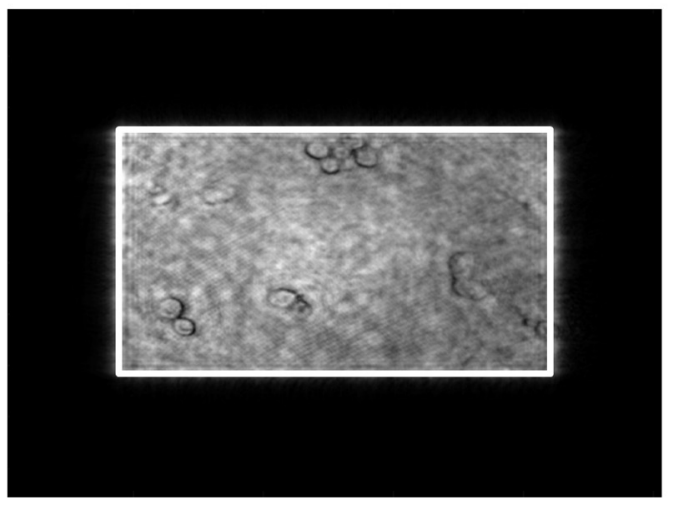

(c)

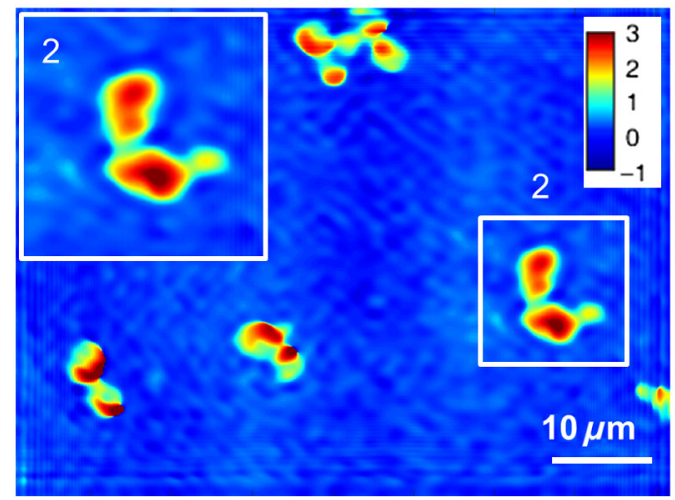

(e)

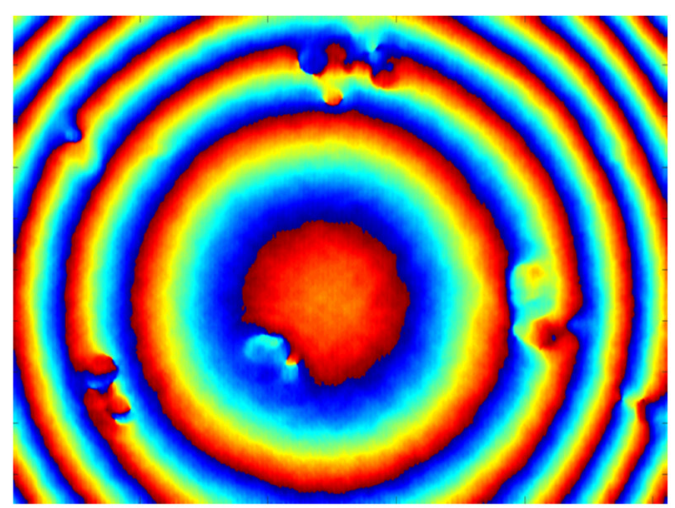

(b)

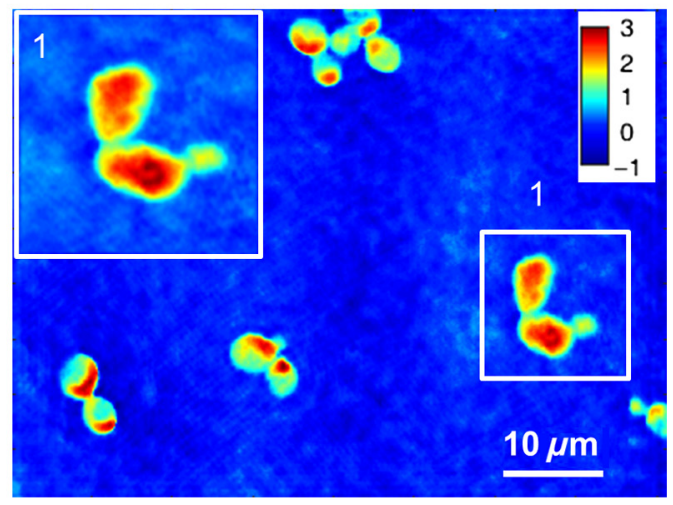

(d)

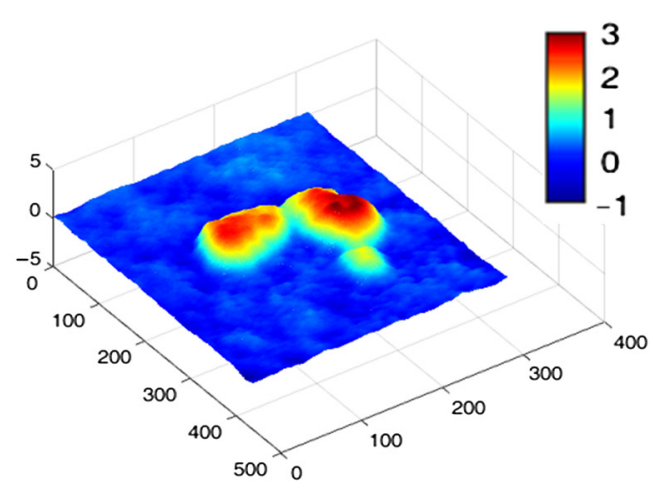

(f)

Fig. 5 Imaging of yeast cells using constrained optimization approach on DHM. The results are compared with reconstruction using Fourier filter method [Fig. 5(e)]. (a) Amplitude image and (b) phase of the recovered complex object wave. (c) Amplitude of object at the image plane obtained by Fresnel propagation. (d) Aberration-corrected phase image at the image plane. (e) Aberration-corrected phase image at the image plane using Fourier filter method of filter size $40 \times 40$ pixels. Inset in (d) and (f) show the zoomed view of rectangle marked as 1 and 2. (f) 3-D rendering of yeast cell for the rectangle shown in (d) (color bar indicates phase in radians). 


$$
\Gamma(m, n)=\exp \left[-i \sum_{\alpha=0}^{O} P_{\alpha} Z^{\alpha}\right]
$$

where $P_{\alpha}$ is the coefficient of the Zernike polynomial $\left(Z^{\alpha}\right)$ and $O$ is the order of the polynomial. Finally, the aberration-corrected complex image $\psi(m, n)$ in the image plane is obtained by Eq. (14). More details of the procedure are explained in Refs. 32 and 33.

\section{Results and Discussions}

We have tested the constrained optimization approach for imaging live yeast cells. Yeast cells were grown in a yeast peptone dextrose medium, and $2 \mu \mathrm{L}$ of the culture were pipetted out onto a microscopic glass slide and covered with coverslip. The hologram is recorded using the MO $100 \times$ and $\mathrm{NA}=0.95$. A near on-axis angle of $\sim 0.6 \mathrm{deg}$ is used between the object beam and the reference beam. This angle is well below the minimum angle criterion, hence making it difficult to separate DC and twin image terms by Fourier filtering. Hence in this system, Fourier filtering will decide the maximum achievable resolution that is lower than the optical diffraction limit of the system. In this paper, we compare the reconstruction of a hologram by both the Fourier filtering method and the constrained optimization method. The recorded hologram and amplitude of Fourier transform of the hologram are shown in Figs. 3(a) and 3(b), respectively.

\subsection{Image Recovery by Fourier Filtering Approach}

Fourier filtering is a well-known method for separating the DC and twin images from the off-axis hologram. Three different sizes of Fourier filters were used to demonstrate the quality of reconstruction by this method. We filtered the $\mathrm{OR}^{*}$ term for the reconstruction process. In the amplitude of Fourier transform of the hologram image as shown in Fig. 3(b), the distance between the DC and the sideband peak is 118 pixels. Initially, we started with the Fourier filter of size of $40 \times 40$ pixels centered on the OR* peak as shown in Fig. 4(a). The reconstructed amplitude and phase images of the Fourier filtered hologram are shown in Figs. 4(b) and 4(c), respectively. The phase images shown are aberration-corrected and details of the aberration correction is shown in Sec. 3.5. The object region marked as a white rectangle in the amplitude images is used for aberration correction. The reconstructed image does not contain any DC or twin image term but the resolution is poor because of the selection of a low number of pixels in filtering. In the next step, we used a Fourier filter of size $120 \times 120$ pixels as shown in Fig. 4(d). Now, the reconstructed amplitude and phase images have improved in resolution as shown in Figs. 4(e) and 4(f), respectively, but it has interference noise from the background DC that destroys the original resolution. Similarly as the size of the Fourier filter increases to $240 \times 240$ pixels as shown in Fig. 4(g), the reconstructed amplitude and phase images contain both DC and OR* terms as shown in Figs. 4(h) and 4(i), respectively. Even though the resolution of the reconstructed image increased with a larger filter size, the presence of strong DC has increased the noise thereby spoiling the achievable resolution completely.

\subsection{Image Recovery by Solving Minimum L2-Norm Problem with Smoothness Constraint}

We used the constrained optimization approach to reconstruct the same hologram that was used for Fourier filtered

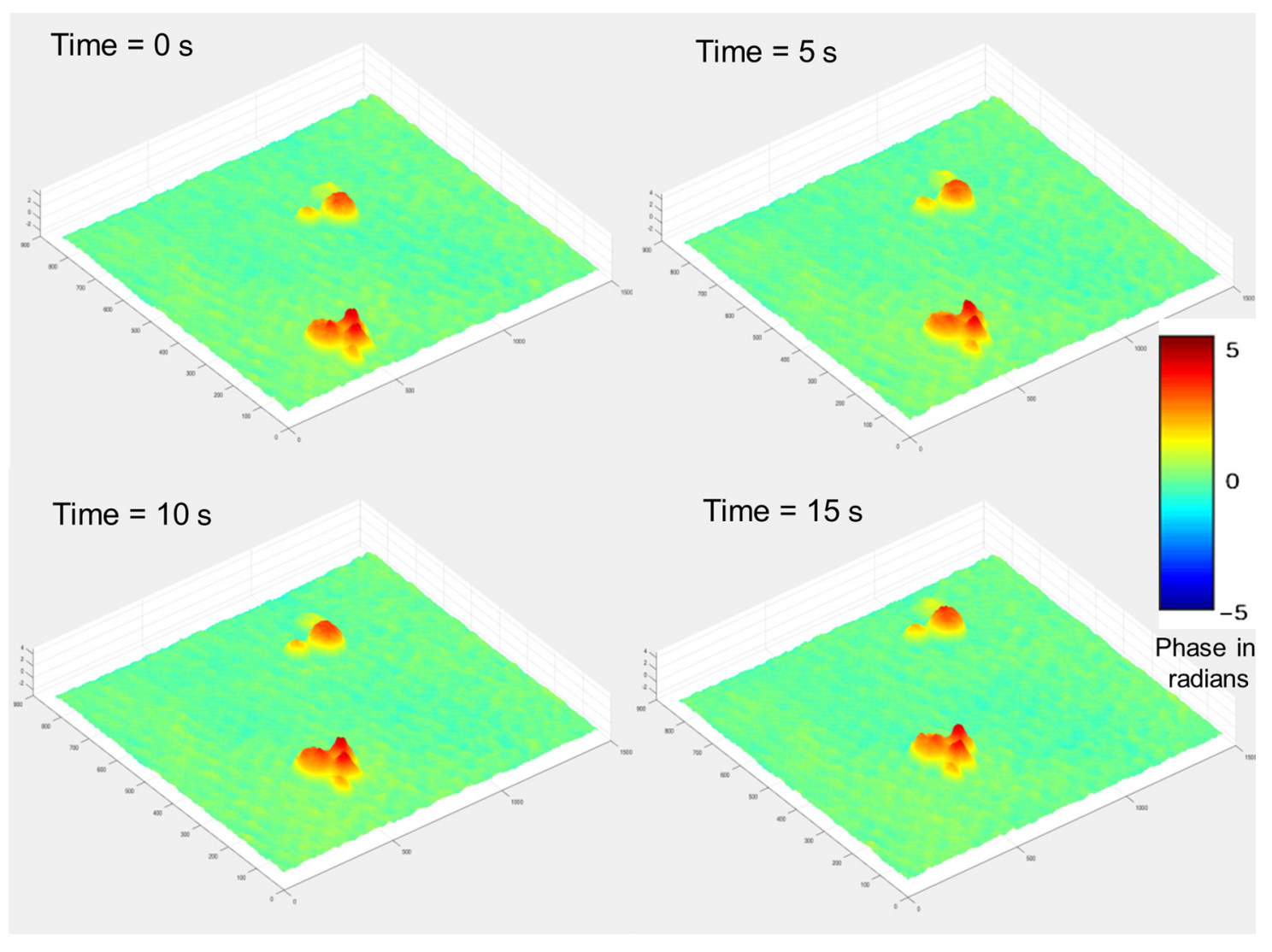

Fig. 6 Dynamics of live dividing yeast cells with different times stamps (color bar indicates phase in radians) (Video 1, QuickTime 10.9 MB [URL: http://dx.doi.org/10.1117/1.JBO.21.10.106003.1]). 
reconstruction. The solution to the optimization algorithm [Eq. (9)] is an iterative approach solved for minimum L2 norm. This approach provides the complex object wave in the hologram plane from a single hologram. The reference beam is numerically coded by Eq. (2) to exactly match the experimental reference beam. The distance between DC and sidebands in the $x$-direction is 118 pixels and the $y$-direction is 0 pixels. With the wavelength of $532 \mathrm{~nm}$ and pixel size of $2.2 \mu \mathrm{m}$, the value of $k_{x}$ and $k_{y}$ is found to be 0.0111 and 0 , respectively. We used a zero matrix of the size of object as an initial guess for this iterative approach. In our case, the solution converged to a minimum L2 norm error in 30 iterations. The step size " $\alpha$," which is in the range of 0.01 to 0.1 , was selected using the line-search method.
The solution to Eq. (6) is modulated by the carrier frequencies and its harmonics. This modulation can be filtered by an averaging filter $(G)$ in each iteration. The size of the averaging filter is chosen in such a way that it should be half of the fringe period of the hologram. In our case, the hologram fringe period was 24 pixels and hence the size of the average filter was $12 \times 12$ pixels. It may be noted that this averaging filter has a slight smoothening effect on the sharp edges of the object. Hence, we observed that the recording of the hologram at a short defocus distance from the image plane helped in recovering the sharp edges of the object better. The calculated computation time for 30 iterations is $15 \mathrm{~s}$ on a PC with $3.4 \mathrm{GHz}$ Intel core processor. A graphics processing unit-based computation

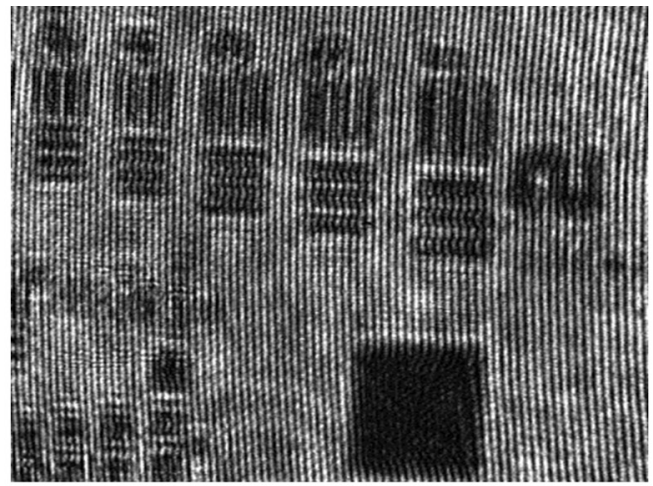

(a)

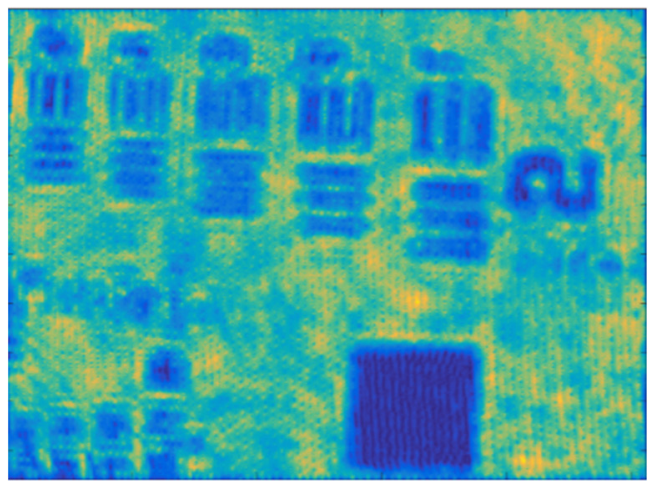

(c)

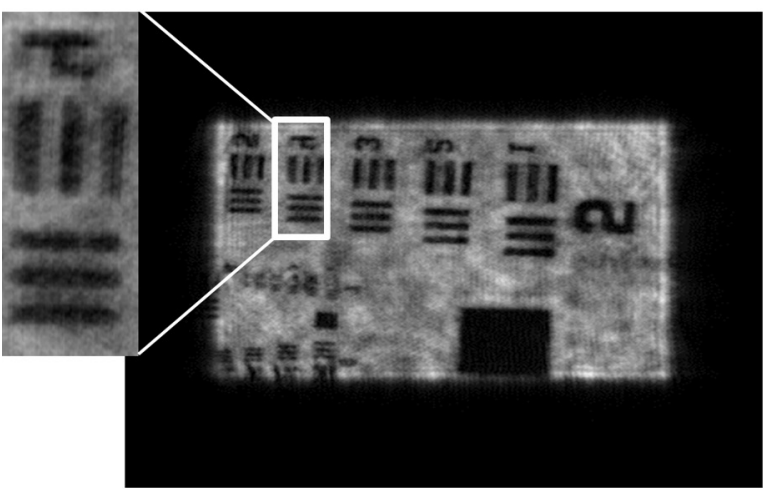

(e)

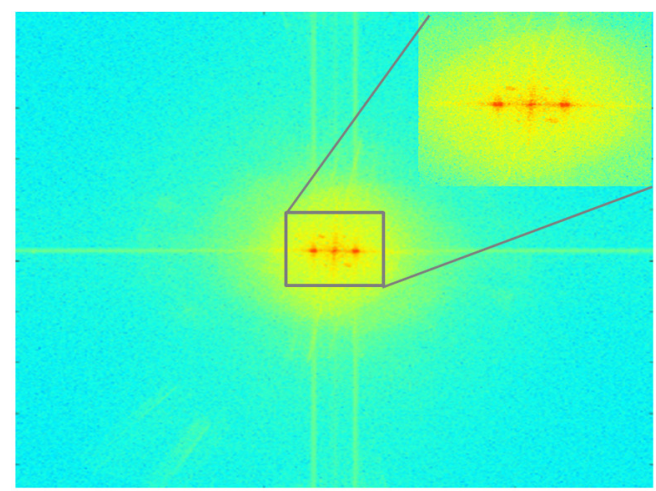

(b)

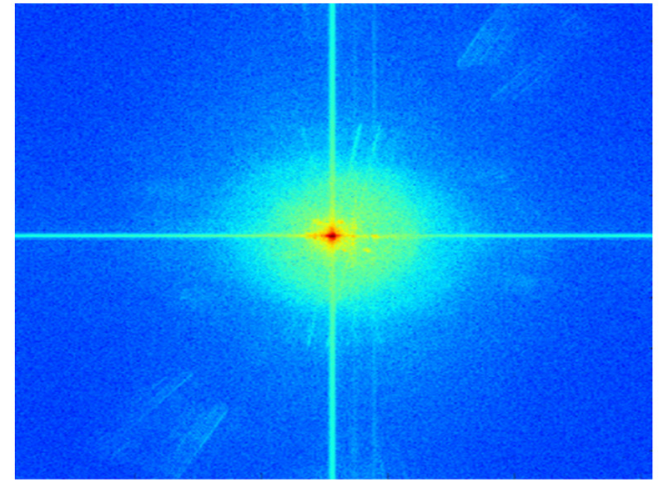

(d)

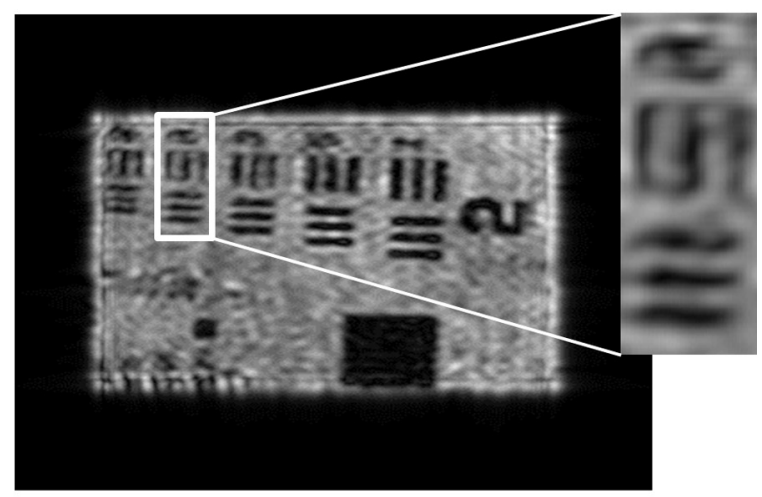

(f)

Fig. 7 Comparsion of constrained optimization approach and Fourier filtering approach. (a) Hologram of USAF resolution target. (b) Amplitude of Fourier transform of the hologram. (c) Amplitude image of the recovered complex object wave. (d) Amplitude of the Fourier transform of recovered object wave. (e) Reconstructed amplitude image using constrained optimization approach. (f) Reconstructed amplitude image using Fourier filtering approach of filter size $40 \times 40$ pixels. 
with further code optimization would further decrease the computation time. Figures 5(a) and 5(b) show the amplitude and phase of recovered object field in the hologram plane. The microscopic objective used in the object arm produces a wave front curvature in the object beam and affects only the phase of image. This curvature is clearly visible in the phase image [Fig. 5(b)]. The recovered complex object plane is back-propagated to the image plane by Fresnel back propagation [Eq. (10)] and the amplitude of the object field is shown in Fig. 5(c). The aberrated-phase image is numerically corrected by the algorithm mentioned in Sec. 3.5 and only the object region [marked as a white rectangle in Fig. 5(c)] is used for phase correction. A Zernike polynomial of order 21 was used for aberration correction. The aberration-corrected phase image is shown in Fig. 5(d).
Comparison of the reconstructed amplitude images [Figs. 4(b) and 5 (c) of the Fourier filtering of size $40 \times 40$ pixels and constrained optimization approach, respectively] shows that our approach provides images with improved resolution and less speckle noise and without any interference from DC and twin image terms. The phase images retrieved through the constrained optimization approach [Fig. 5(d)] show clear subcellular features [zoomed view show in inset] compared to the phase images obtained through the Fourier filtering approach [Fig. 5(e)] with a filter size of $40 \times 40$ pixels. Three-dimensional (3-D) rendering of yeast cells for the rectangle 1 shown in Fig. 5(d) is shown in Fig. 5(f). The thickness of yeast cells was found to be in the range of 3 to $5 \mu \mathrm{m}$ with the average refractive index as 1.395 (Refs. 35 and 36) and surrounding medium had a

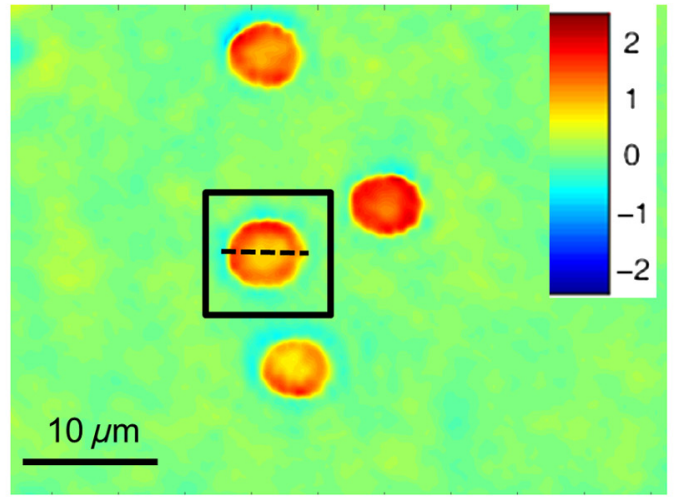

(a)

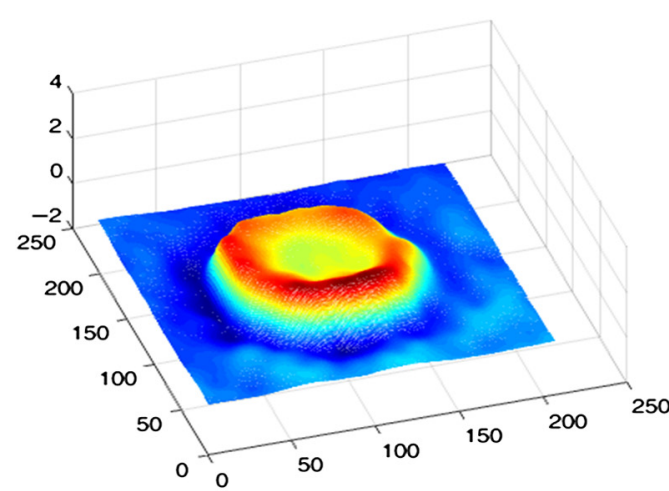

(c)

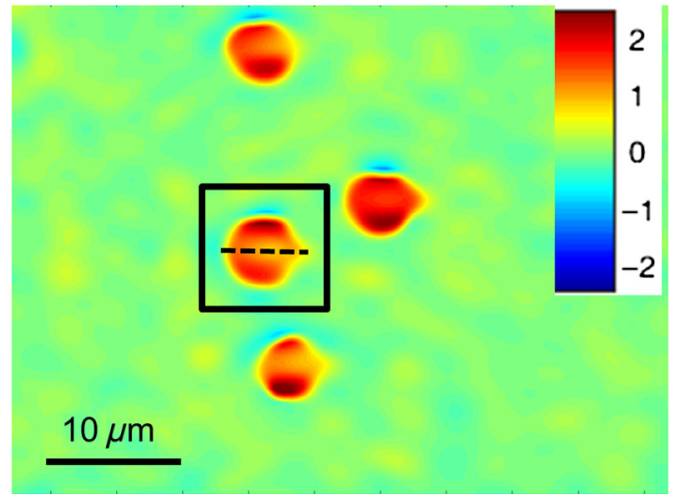

(b)

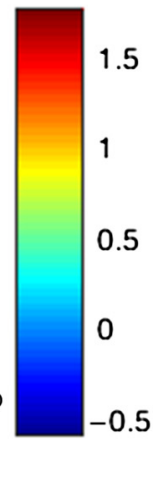

1.5

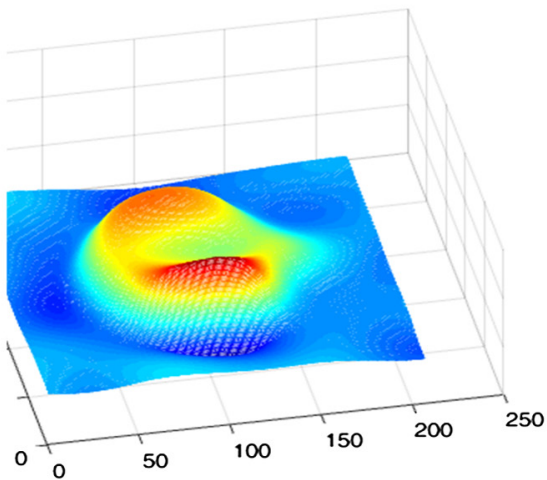

(d)

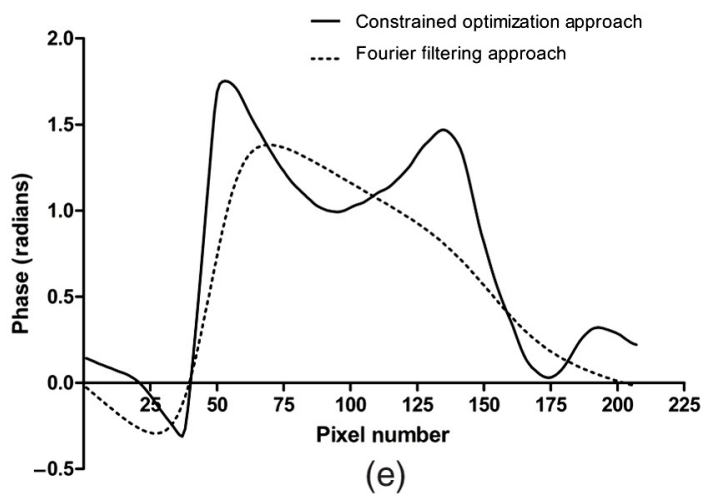

Fig. 8 Reconstructed phase image of RBCs (a) and (c) constrained optimization approach (b) and (d) Fourier filtering approach. (c) and (d) 3-D rendering of RBC marked as rectangle in (a) and (b), respectively. (e) Line profile across cells in (a) and (b) (dotted line) indicating the improvement in phase resolution in the constrained optimization approach. (Color bar indicates phase in radians.) 
refractive index of 1.339 . QPI of actively dividing live yeast cells were carried out to demonstrate the capability of the system for observing the real-time dynamics using single-shot imaging. The holograms of live yeast cells were recorded at the rate of 4.4 frames/s for $22 \mathrm{~s}$. The holographic videos were recorded in real time and reconstructed offline with our optimization approach. The phase images of live dividing yeast cells at different time stamps are shown in Fig. 6 and the multimedia file is shown in Video 1.

A USAF resolution chart was used as an object to demonstrate the resolution improvement. An MO $(10 \times, \mathrm{NA}=0.25)$ was used for recording the hologram. The recorded hologram and amplitude of the Fourier transform of the hologram are shown in Figs. 7(a) and 7(b), respectively. Figures 7(c) and 7(d) show the amplitudes of the recovered object field and the Fourier transform of the recovered object field in the hologram plane using the optimization approach. The reconstructed amplitude image [Fig. 7(e)] at the image plane is obtained by Fresnel back propagation [Eq. (10)] of the recovered complex object field. Reconstructed amplitude image [Fig. 7(f)] using the Fourier filtering of size $40 \times 40$ pixels is shown here for comparison. Insets in Figs. 7(e) and 7(f) show the zoomed view of the group 5 element 4 of the USAF resolution target in both the optimization approach and the conventional Fourier filtering approach, respectively. This shows that objects can be clearly recovered with improved resolution using a constrained approach from a near on-axis hologram from a single shot.

We also demonstrate this approach by reconstructing the near on-axis holograms recorded for RBCs. RBCs are prepared for imaging according to the standard procedure. ${ }^{37}$ Two micro liters of diluted RBCs were pipetted out on the glass slide and covered with the cover slip. The holograms are recorded with MO of $100 \times$ and NA $=0.95$. The reconstructed phase images of RBCs with our constrained optimization approach and conventional Fourier transform approach are shown in Figs. 8(a) and 8(b), respectively. 3-D renderings of RBC shown in rectangles in Figs. 8(a) and 8(b) are shown in Figs. 8(c) and 8(d). The line profiles across the single RBC in Figs. 8(a) and 8(b) (dotted line) are shown in Fig. 8(e). Due to the overlapping DC and $\mathrm{OR}^{*}$ term and subsequent limitation in selection of spatial frequencies by Fourier filter window size, the biconcave shape could not be reconstructed using the Fourier filtering approach, whereas the constrained optimization approach could reconstruct the biconcave shape of RBC.

By performing a near on-axis recording with overlapping interference terms, we capture higher spatial frequencies that would have been lost otherwise. Hence, we expect improved resolution in this technique in cases in which an off-axis technique would lose the higher spatial frequencies and result in lower resolution due to the angle chosen for the reference beam. As the constrained optimization approach can effectively retrieve the object in the presence of overlapping DC and twin image terms, we can effectively exploit the maximum spacebandwidth in terms of camera pixels and collected spatial frequencies without having to worry about overlapping terms. The proof of concept is well evident in the results reported using the USAF resolution chart [Figs. 7(e) and 7(f)], RBCs [Figs. 8(b) and $8(\mathrm{c})$ ], showing improved resolution using the current technique compared to the Fourier filtering approach.

\section{Conclusion}

We demonstrate a quantitative phase microscope with a constrained optimization approach, capable of recovering images using a USAF resolution target, RBCs, and yeast cells. DHM with this optimization approach provides high-resolution image recovery even if a minimum reference beam criterion is not met to separate DC and twin image. The small reference beam angle is automatically calculated with a single hologram without any additional recording. Compared to conventional Fourier filtering DHM, this system has the ability to provide the images with improved resolution allowed by a digital detector array with a single shot. We also demonstrated an automatic aberration correction algorithm to correct the phase aberrations introduced by MO without any additional objectives in the reference beam. The results reported here demonstrate the strong potential of DHM in imaging live cells in three dimensions and their dynamics in real time with high sensitivity and high resolution. The use of a constrained optimization approach is promising for delivering a compact platform with near-zero angle between object and reference beams and with effective utilization of the camera resolution rendering high-resolution images at a slightly higher computational cost. This high-resolution computational approach for image recovery has high potential in rapid clinical quantifications and pathological applications in the near future.

\section{Acknowledgments}

One of the authors, Renu John, acknowledges financial support from the Science and Engineering Research Board (SERB), DST, India (SR/S2/LOP-0024/2012) and BRNS, India (34/ 14/04/2015/BRNS).

\section{References}

1. F. Zernike, "Phase contrast, a new method for the microscopic observation of transparent objects," Physica 9(7), 686-698 (1942).

2. Z. Wang et al., "Spatial light interference microscopy (SLIM)," Opt. Express 19, 1016-1026 (2011).

3. I. Yamaguchi and T. Zhang, "Phase-shifting digital holography," Opt. Lett. 22(16), 1268-1270 (1997).

4. T. Ikeda et al., "Hilbert phase microscopy for investigating fast dynamics in transparent systems," Opt. Lett. 30(10), 1165-1167 (2005).

5. X. Yu et al., "Review of digital holographic microscopy for threedimensional profiling and tracking," Opt. Eng. 53(11), 112306 (2014).

6. X. Yu et al., "Four-dimensional motility tracking of biological cells by digital holographic microscopy," J. Biomed. Opt. 19(4), 045001 (2014).

7. Y. Park et al., "Diffraction phase and fluorescence microscopy," Opt. Express 14(18), 8263-8268 (2006).

8. G. Popescu et al., "Diffraction phase microscopy for quantifying cell structure and dynamics," Opt. Lett. 31(6), 775-777 (2006).

9. E. Shaffer et al., "Digital holographic microscopy investigation of second harmonic generated at a glass/air interface," Opt. Lett. 34(16), 2450-2452 (2009).

10. N. Pavillon et al., "Study of intracellular ion dynamics with a multimodality approach combining epifluorescence and digital holographic microscopy," in OSA Technical Digest (CD), Biomedical Optics and 3-D Imaging JMA26 (2010).

11. Y. Cotte et al., "Marker-free phase nanoscopy," Nat. Photonics 7(2), 113-117 (2013).

12. P. Marquet et al., "Exploring neural cell dynamics with digital holographic microscopy," in OSA Technical Digest (online), Digital Holography and Three-Dimensional Imaging DM4A.1 (2013).

13. G. Popescu et al., "Erythrocyte structure and dynamics quantified by Hilbert phase microscopy," J. Biomed. Opt. 10(6), 060503 (2005).

14. N. Lue et al., "Tissue refractometry using Hilbert phase microscopy," Opt. Lett. 32(24), 3522-3524 (2007).

15. P. Marquet et al., "Digital holographic microscopy: a noninvasive contrast imaging technique allowing quantitative visualization of living cells with subwavelength axial accuracy," Opt. Lett. 30(5), 468-470 (2005). 
16. G. D. Caprio et al., "Quantitative label-free animal sperm imaging by means of digital holographic microscopy," IEEE J. Sel. Top. Quantum Electron. 16(4), 833-840 (2010).

17. I. Crha et al., "Digital holographic microscopy in human sperm imaging," J. Assisted Reprod. Genet. 28(8), 725-729 (2011).

18. B. Bhaduri et al., "Diffraction phase microscopy with white light," Opt. Lett. 37(6), 1094-1096 (2012).

19. J. W. Goodman, Introduction to Fourier Optics, Roberts \& Company, Pittsburgh, Pennsylvania (2005).

20. M. Liebling, T. Blu, and M. Unser, "Complex-wave retrieval from a single off-axis hologram," J. Opt. Soc. Am. A 21(3), 367-377 (2004).

21. D. Carl et al., "Parameter-optimized digital holographic microscope for high-resolution living-cell analysis," Appl. Opt. 43(36), 6536-6544 (2004).

22. E. Cuche, P. Marquet, and C. Depeursinge, "Spatial filtering for zeroorder and twin-image elimination in digital off-axis holography," Appl. Opt. 39(23), 4070-4075 (2000).

23. M. K. Kim, "Principles and techniques of digital holographic microscopy," Proc. SPIE 1(1), 018005 (2010).

24. U. Schnars and W. Jüptner, Digital Holography: Digital Hologram Recording, Numerical Reconstruction, and Related Techniques, Springer, New York (2005).

25. K. Khare, P. T. S. Ali, and J. Joseph, "Single shot high resolution digital holography," Opt. Express 21(3), 2581-2591 (2013).

26. P. T. Samsheerali, K. Khare, and J. Joseph, "Quantitative phase imaging with single shot digital holography," Opt. Commun. 319, 85-89 (2014).

27. M. Singh and K. Khare, "Single shot high resolution digital holographic microscopy," in 12th Int. Conf. on Fiber Optics and Photonics, Paper M4A.3 (2014).

28. E. Abbe, "Beiträge zur Theorie des Mikroskops und der mikroskopischen Wahrnehmung," Archiv für mikroskopische Anatomie 9(1), 413-418 (1873).

29. V. P. Pandiyan, K. Khare, and R. John, "High resolution quantitative phase imaging of live cells with constrained optimization approach," Proc. SPIE 9718, 971803 (2016).

30. A. K. Jain, Fundamentals of Digital Image Processing, Prentice-Hall, Inc., Upper Saddle River, New Jersey (1989).

31. E. Cuche, P. Marquet, and C. Depeursinge, "Simultaneous amplitudecontrast and quantitative phase-contrast microscopy by numerical reconstruction of Fresnel off-axis holograms," Appl. Opt. 38(34), 6994-7001 (1999).
32. T. Colomb et al., "Automatic procedure for aberration compensation in digital holographic microscopy and applications to specimen shape compensation," Appl. Opt. 45(5), 851-863 (2006).

33. T. Colomb et al., "Numerical parametric lens for shifting, magnification, and complete aberration compensation in digital holographic microscopy," J. Opt. Soc. Am. A 23(12), 3177-3190 (2006).

34. V. P. Pandiyan and R. John, "Optofluidic bioimaging platform for quantitative phase imaging of lab on a chip devices using digital holographic microscopy," Appl. Opt. 55(3), A54-A59 (2016).

35. F. D. Bryant, P. Latimer, and B. A. Seiber, "Changes in total light scattering and absorption caused by changes in particle conformation-a test of theory," Arch. Biochem. Biophys. 135, 109-117 (1969).

36. B. E. Pyle, A. Brunsting, and P. Latimer, "Detection of the vacuole of yeast cells in suspension by transmittance radiometry," Appl. Opt. 18(21), 3615-3619 (1979).

37. G. Popescu et al., "Optical measurement of cell membrane tension," Phys. Rev. Lett. 97, 1-4 (2006).

Vimal Prabhu Pandiyan received his BTech degree in biomedical engineering and his MTech degree in medical electronics from Anna University, Chennai, Tamil Nadu, India, in 2006 and 2011, respectively. $\mathrm{He}$ is currently pursuing his $\mathrm{PhD}$ in biomedical engineering at the Indian Institute of Technology Hyderabad. His current research interests are digital holographic microscopy, coherence and lowcoherence microscopy, and quantitative phase imaging.

Kedar Khare received his $\mathrm{PhD}$ from the Institute of Optics, University of Rochester, New York, USA, in 2004. After spending a couple years more at the University of Rochester as a research associate and over five years at GE Global Research as a lead scientist, he has been a faculty member in the Department of Physics, Indian Institute of Technology Delhi, since December 2011. His research interests include optics/photonics, computational imaging, inverse problems, signal processing, and compressive sensing.

Renu John received his $\mathrm{PhD}$ from the Indian Institute of Technology Delhi in 2006. He carried out his postdoctoral research at the Fitzpatrick Center for Photonics, Duke University, Durham, North Carolina, USA, and Biophotonics Imaging Laboratory, University of Illinois, Urbana-Champaign, USA. He is currently an associate professor and head of the Department of Biomedical Engineering, Indian Institute of Technology Hyderabad. His research interests include nanobiophotonics, noninvasive NIR bio-imaging, 3-D phase microscopy, and optical coherence tomography (OCT). 